\title{
Decay constants and spectroscopy of mesons in lattice QCD using domain-wall fermions
}

\author{
JLQCD Collaboration: B. Fahy ${ }^{\star a \dagger}$, G. Cossu $^{a}$, S. Hashimoto ${ }^{a, b}$, T. Kaneko ${ }^{a, b}$, J. \\ Noaki $^{a}$, M. Tomii ${ }^{b}$ \\ ${ }^{a}$ High Energy Accelerator Research Organization (KEK), Ibaraki 305-0801, Japan Address \\ ${ }^{b}$ Department of Particle and Nuclear Science, SOKENDAI (The Graduate University for \\ Advanced Studies), Ibaraki 305-0801, Japan
}

\begin{abstract}
We report results of masses and decay constants of light and charmed pseudo-scalar mesons using lattice QCD with Möbius domain-wall fermions. Using this formulation we are able to compute pseudo-scalar decay constants through the pseudo-scalar density operator as well as with the axial-vector current. Results are shown from several lattice spacings and pion masses between $230 \mathrm{MeV}$ and $500 \mathrm{MeV}$. We present an analysis of these results at different quark masses to show the chiral properties of the light mesons masses and decay constants.
\end{abstract}

The 33rd International Symposium on Lattice Field Theory

14 - 18 July 2015

Kobe International Conference Center, Kobe, Japan*

\footnotetext{
${ }^{*}$ Speaker.

†Email:bfahy@post.kek.jp
} 


\section{Introduction}

The JLQCD collaboration has recently produced ensembles of lattice configurations with fine lattice spacings and good chiral symmetry. Lattice simulations of 2+1-flavor QCD were performed using the Möbius domain-wall fermions [1] with tree-level Symanzik gauge action. Table 1 shows the 15 gauge ensembles generated [2]. These lattices have lattice spacings of $1 / a \approx 2.4,3.6$, and 4.5 GeV with pion masses between $230 \mathrm{MeV}$ and $500 \mathrm{MeV}$. For each ensemble, 10, 000 molecular dynamics (MD) times were run after thermalization.

Using domain-wall fermions the Ginsparg-Wilson relation is only approximate. The violation of the Ginsparg-Wilson relation is given by the residual mass. The Möbius representation as well as using stout link-smearing [3] make the residual mass small of $\mathscr{O}(1 \mathrm{MeV})$ on the coarsest ( $\beta=4.17$ ) lattices and $<0.2 \mathrm{MeV}$ on the finer lattices [4]. Good chiral symmetry enables simpler renormalization such as $Z_{V}=Z_{A}$, and simplifies the calculation of the pseudo-scalar decay constants directly utilizing the PCAC relation.

With chiral symmetry preserved, observables can be used to compare lattice QCD results to those of Chiral Perturbation Theory (ChPT). In this work we present results of masses and decay constants of light and charmed pseudo-scalar mesons. These measurements are used to determine the low energy constants (LEC) in ChPT. Additionally the fine lattice spacing enables exploration of charm physics with manageable cutoff effects.

In this work the scale for these lattices was determined from Wilson flow using $t_{0}$. We use the value from [5] as an input. The determination was done using a linear extrapolation to the physical point in $m_{\pi}^{2}$ as well as an interpolation of the strange quark mass to reproduce the physical $\left(M_{s \bar{s}}^{\text {phys }}\right)^{2}=2 M_{K}^{2}-M_{\pi}^{2}$. The fitted parameters to describe the mass dependence for the two smaller $\beta$ values were then used to determine the scale on our finest lattice. The results for $a^{-1}$ on each $\beta$ are listed in Table 1.

\section{Computation of observables}

Pseudo-scalar correlators were produced utilizing our QCD software package Iroiro++ [6]. These correlators were computed on 200 gauge configurations separated by $50 \mathrm{MD}$ times and from two source locations, producing 400 measurements of the light correlators and 300 measurements of heavy correlators for each ensemble, except for the $\beta=4.17$ ensemble on the larger volume, which has 600 light and 400 heavy measurements. Correlators were produced with unsmeared point sources as well as smeared sources using Gaussian smearing, and the same point and smeared operators are used also for the sinks. Gaussian smearing is defined by the operator $(1-(\alpha / N) \Delta)^{N}$ where $\Delta$ as the Laplacian and in this work the parameters $\alpha=20.0$ and $N=200$ were used.

The amplitudes of the unsmeared local operators are required to compute the decay constants. Two-point correlation functions of the form $\left\langle P^{L}(x) P^{G^{\dagger}}(0)\right\rangle$ were fit simultaneously with correlators $\left\langle P^{G}(x) P^{G^{\dagger}}(0)\right\rangle$ where $L$ indicates an unsmeared local operator while $G$ denotes Gaussian smeared operators. The two-point correlation functions were fit to the functional form

$$
C=\underbrace{\frac{1}{2 m_{\pi}}\langle 0|P| \pi\rangle\left\langle\pi\left|P^{\dagger}\right| 0\right\rangle}_{A_{P P}}\left(e^{-m_{\pi} t}+e^{-m_{\pi}\left(N_{t}-t\right)}\right)
$$




\begin{tabular}{|l|l|r|r|r|r|r|}
\hline Lattice Spacing & $L^{3} \times T$ & $L_{5}$ & $a m_{u d}$ & $a m_{s}$ & $m_{\pi}[\mathrm{MeV}]$ & $m_{\pi} L$ \\
\hline$\beta=4.17$, & $32^{3} \times 64(L=2.6 \mathrm{fm})$ & 12 & 0.0035 & 0.040 & 230 & 3.0 \\
$a^{-1}=2.453(4) \mathrm{GeV}$ & & & 0.0070 & 0.030 & 310 & 4.0 \\
& & & 0.0070 & 0.040 & 310 & 4.0 \\
& & & 0.0120 & 0.030 & 400 & 5.2 \\
& & & 0.0120 & 0.040 & 400 & 5.2 \\
& & & 0.0190 & 0.030 & 500 & 6.5 \\
& & & 0.0190 & 0.040 & 500 & 6.5 \\
\cline { 2 - 7 } & $48^{3} \times 96(L=3.9 \mathrm{fm})$ & 12 & 0.0035 & 0.040 & 230 & 4.4 \\
\hline$\beta=4.35$, & $48^{3} \times 96(L=2.6 \mathrm{fm})$ & 8 & 0.0042 & 0.018 & 300 & 3.9 \\
$a^{-1}=3.610(9) \mathrm{GeV}$ & & & 0.0042 & 0.025 & 300 & 3.9 \\
& & & 0.0080 & 0.018 & 410 & 5.4 \\
& & & 0.0080 & 0.025 & 410 & 5.4 \\
& & & 0.0120 & 0.018 & 500 & 6.6 \\
& & & 0.0120 & 0.025 & 500 & 6.6 \\
\hline$\beta=4.47$, & $64^{3} \times 128(L=2.7 \mathrm{fm})$ & 8 & 0.0030 & 0.015 & 280 & 4.0 \\
$a^{-1}=4.496(9) \mathrm{GeV}$ & & & & & & \\
\hline
\end{tabular}

Table 1: Parameters of the JLQCD gauge ensembles used in this work. Pion masses are rounded to the nearest $10 \mathrm{MeV}$. The ensemble with $m_{\pi} L \approx 3.0$ is excluded in all analysis below to avoid possible finite volume effects.

for large $t$ to determine the masses and amplitudes where $P$ is either $P^{L}$ or $P^{G}$. The matrix element of $\langle 0|P| \pi\rangle$ of the unsmeared operator $P^{L}$ can be reconstructed from the simultaneous fit of $\left\langle P^{L}(x) P^{G}(0)\right\rangle$ and $\left\langle P^{G}(x) P^{G}(0)\right\rangle$. The decay constants are calculated by utilizing the axial WardTakahashi identity $Z_{A} \partial_{\mu} A_{\mu}=\left(m_{q_{1}}+m_{q_{2}}\right) P$, where $A_{\mu}$ is the lattice axial current, and $m_{q}$ 's are the quark masses of the pseudo-scalar meson of interest. This leads to the formula for $f_{P}$,

$$
f_{P}=\left(m_{q_{1}}+m_{q_{2}}\right) \sqrt{\frac{2 A_{P P}}{m_{\pi}^{3}}},
$$

which does not rely on the renormalization constant $Z_{A}$. The mass $m_{q}$ used is the bare quark mass plus the residual mass. We use the convention $F_{\pi}=f_{\pi} / \sqrt{2}$.

\section{Pion masses and decay constants}

Our measurements of the pion masses and decay constants for ensembles at different bare light quark masses allow us to investigate the consistency with $S U(2) \mathrm{ChPT}$. The quark mass dependence of $M_{\pi}$ and $F_{\pi}$ at next-to-next-to-leading order [7] is

$$
\begin{aligned}
\frac{M_{\pi}^{2}}{\bar{m}_{q}} & =2 B\left[1-\frac{1}{2} x \ln \frac{\Lambda_{3}^{2}}{M^{2}}+\frac{17}{8} x^{2}\left(\ln \frac{\Lambda_{M}^{2}}{M^{2}}\right)^{2}+k_{M} x^{2}+\mathscr{O}\left(x^{3}\right)\right], \\
F_{\pi} & =F\left[1+x \ln \frac{\Lambda_{4}^{2}}{M^{2}}-\frac{5}{4} x^{2}\left(\ln \frac{\Lambda_{F}^{2}}{M^{2}}\right)^{2}+k_{F} x^{2}+\mathscr{O}\left(x^{3}\right)\right] .
\end{aligned}
$$



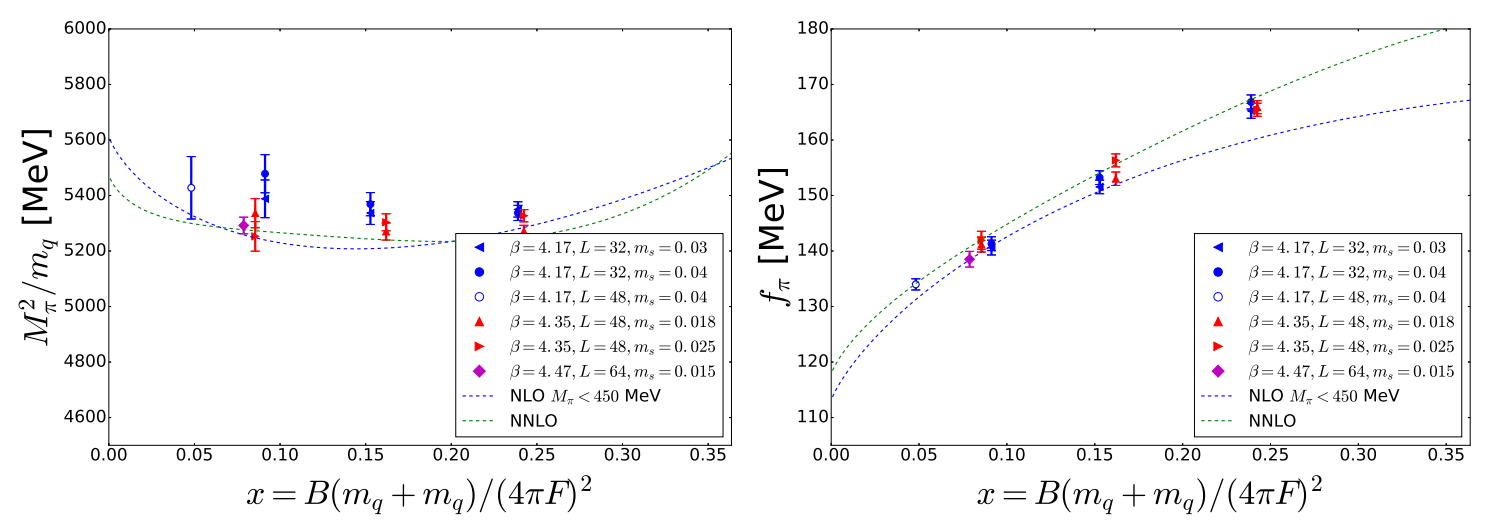

Figure 1: Plots of $M_{\pi}^{2} / \bar{m}_{q}$ (left panel) and $F_{\pi}$ (right panel), both vs. $x=2 \bar{m}_{q} B /(4 \pi F)^{2}$. Fit lines show the best NLO (blue) and NNLO (green) fits in the continuum and physical strange quark mass limits. The NLO fits only include the ensembles for $M_{\pi}<450 \mathrm{MeV}$


Figure 2: Same as Figure 1 except plotted vs. $\xi=M_{\pi}^{2} /\left(4 \pi F_{\pi}\right)^{2}$.

These are expanded using the parameter $x=M^{2} /(4 \pi F)^{2}$ where $M^{2}=B\left(\bar{m}_{q}+\bar{m}_{q}\right)=2 \bar{m}_{q} \Sigma / F^{2}$. $\bar{m}_{q}$ is the appropriately renormalized quark mass, where the renormalization factor is discussed in [8]. The parameters $\Lambda_{3}$ and $\Lambda_{4}$ are related to the effective coupling constants of ChPT through $\bar{\ell}_{n}=\ln \Lambda_{n}^{2} / M_{\pi}^{2} . \Lambda_{M}$ and $\Lambda_{F}$ are linear combinations of different $\Lambda_{n}$ 's [7].

The chiral expansions above are fit to the data for $F_{\pi}$ and $M_{\pi}^{2} / \bar{m}_{q}$ simultaneously at both NLO and NNLO. At NLO only terms up to $\mathscr{O}\left(x^{2}\right)$ in (3.1) and (3.2) are included leaving the free parameters $F, B, \Lambda_{3}$, and $\Lambda_{4}$. For NNLO there are the additional free parameters $k_{M}$, and $k_{F}$, while the values of $\Lambda_{1}$ and $\Lambda_{2}$ were fixed to the phenomenological value from [9].

To account for the strange-quark mass dependence the fit function was corrected by a term proportional to $M_{s \bar{s}}^{2}=2 M_{K}^{2}-M_{\pi}^{2}$. Combining with a lattice spacing dependence, all fits were performed with a prefactor $\left(1+\gamma_{a} a^{2}+\gamma_{s}\left(M_{s \bar{s}}-M_{s \bar{s}}^{\text {phys }}\right)\right)$. At NLO the fits have $\chi^{2}$ less than 1.5 if including only the ensembles with pion masses $M_{\pi}<450 \mathrm{MeV}$, so the other ensembles were excluded for the NLO fits. For NNLO fits the ensembles of all pion masses were included. The results of the NLO and NNLO fits in the continuum and physical strange quark mass limits are shown in Figure 1 by dashed lines.

Alternatively the ChPT expansions can be reorganized using the parameter $\xi=M_{\pi}^{2} /\left(4 \pi F_{\pi}\right)^{2}$. 
The expansions are

$$
\begin{aligned}
\frac{M_{\pi}^{2}}{\bar{m}_{q}} & =2 B /\left[1+\frac{1}{2} \xi \ln \frac{\Lambda_{3}}{M_{\pi}^{2}}-\frac{5}{8} \xi^{2}\left(\ln \frac{\Omega_{M}^{2}}{M_{\pi}^{2}}\right)^{2}+c_{M} \xi^{2}+\mathscr{O}\left(\xi^{3}\right)\right], \\
F_{\pi} & =F /\left[1-\xi \ln \frac{\Lambda_{4}^{2}}{M_{\pi}^{2}}-\frac{1}{4} \xi^{2}\left(\ln \frac{\Omega_{F}^{2}}{M_{\pi}^{2}}\right)^{2}+c_{F} \xi^{2}+\mathscr{O}\left(\xi^{3}\right)\right],
\end{aligned}
$$

where similarly the values $\Omega_{M}$ and $\Omega_{F}$ are combinations of other LEC's [7]. The pion masses and decay constants are plotted against $\xi$ in Figure 2. The curves represent the fits of NLO and NNLO.

Our preliminary results for the LEC's from the NLO fits expanded in $x$ are $F=83.2(6.3) \mathrm{MeV}$, $\Sigma^{1 / 3}[2 \mathrm{MeV}]=287.9(3.7) \mathrm{MeV}, \bar{\ell}_{3}=3.11(44)$ and $\bar{\ell}_{3}=4.37(22)$. The chiral condensate, $\Sigma$, is renormalized to the one in the $\overline{\mathrm{MS}}$ scheme at $\mu=2 \mathrm{GeV}$ using the renormalization factor calculated in [8]. The values obtained with the two expansion parameters as well as those from NLO and NNLO fits are all consistent within statistical error though the NNLO results have slightly larger uncertainty. $F$ is the decay constant in the chiral limit but at the physical pion mass value we obtain $F_{\pi}=88.9(5.2) \mathrm{MeV}$.

\section{Charmed mesons}

The lattice spacings of the JLQCD ensembles were chosen to treat heavy physics with minimal cutoff effects. We produced charmed correlators using domain-wall heavy quarks at three masses close to the charm mass. All results shown are first interpolated to the charm mass using the spin averaged $c \bar{c}$ masses. Charmonium correlators are also used in the analysis of their time-moments to determine the charm quark mass $m_{c}$ and strong coupling constant $\alpha_{s}$ [10].

Figure 3 shows the masses of the $D$ and $D_{s}$ mesons as well as linear fits in $M_{\pi}^{2}$ accounting for a dependence on the lattice spacing $a^{2}$ and interpolated in $m_{s}$ using $2 M_{K}^{2}-M_{\pi}^{2}$. The raw data for $M_{D_{s}}$ appear scattered because the data points with different input strange quark masses are plotted together. After interpolating in $2 M_{K}^{2}-M_{\pi}^{2}$, the data at different $\beta$ are more consistent with each other. The results after extrapolation are $M_{D}=1867.7(9.5) \mathrm{MeV}$ and $M_{D_{s}}=1964.2(5.0) \mathrm{MeV}$. Their experimental values are $M_{D}^{\text {exp }}=1864.8 \mathrm{MeV}$ and $M_{D_{s}}^{\text {exp }}=1968.3 \mathrm{MeV}$. The dependence upon the lattice cutoff $a$ turned out to be minimal with a difference of $\mathscr{O}(1 \%)$ between the fitted value at $\beta=4.17$ and the continuum limit.

The decay constants of the charmed mesons are also computed using the same process for the pion using the pseudo-scalar current and the appropriate quark masses. These results can be seen in Figure 4. The fitted values after linear extrapolation in $M_{\pi}^{2}$ and $a^{2}$ are $f_{D}=209.6(5.2) \mathrm{MeV}$ and $f_{D_{s}}=244.4(4.1) \mathrm{MeV}$ with the dependence on the lattice spacing turning out to be negligible. For the decay constant of the $D$ meson we attempted to analyze with the ChPT fit at NLO [11] as well as a linear fit. It favors a smaller value for $f_{D}$ because of the chiral logarithm, but more precise data would be necessary to confirm, especially because the current fit is strongly influenced by the lightest data point, which has a relatively large error.

\section{Summary}

We have shown first results from the recently generated JLQCD lattices. The good chiral prop- 

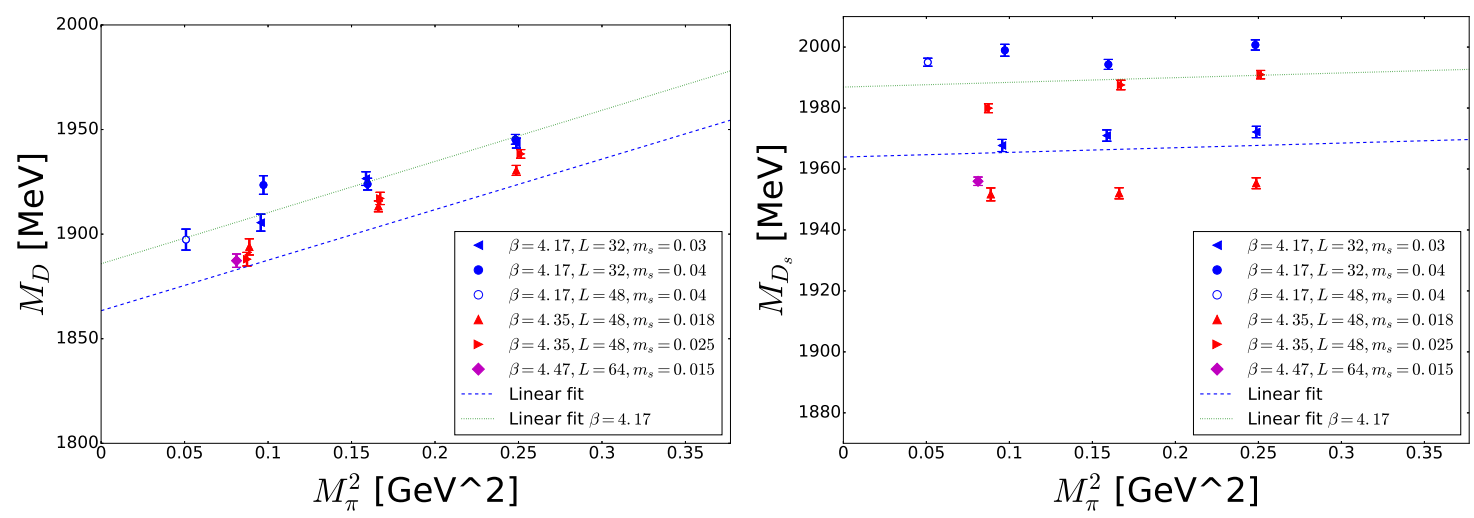

Figure 3: Masses of the $D$ meson (left) and $D_{s}$ meson (right) vs. $M_{\pi}^{2}$. These were fit linearly in $M_{\pi}^{2}$ accounting for a dependence on the lattice spacing $a^{2}$ and interpolated in the strange quark mass using $2 M_{K}^{2}-M_{\pi}^{2}$. The blue dashed line indicated the linear fit extrapolated to the continuum limit while the green dashed line shows the linear fit for the value of $a$ corresponding to our coarsest lattice $\beta=4.17$.
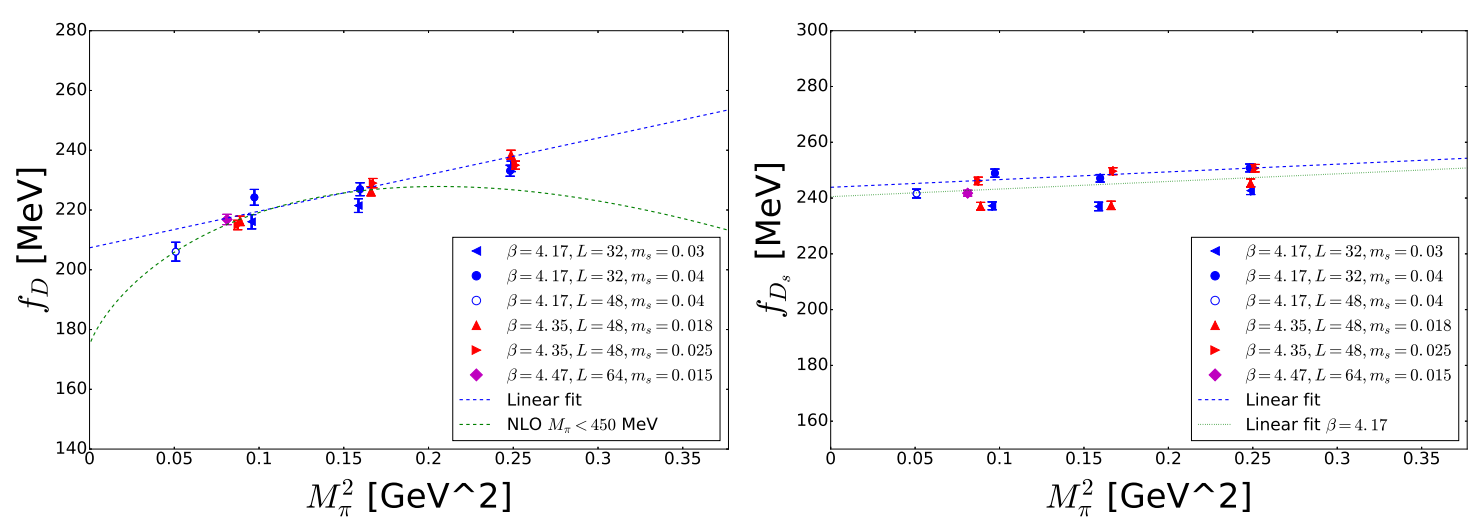

Figure 4: Charmed meson decay constants $f_{D}$ (left panel) and $f_{D_{s}}$ (right panel) vs. $M_{\pi}^{2}$. On both plots the blue dashed line indicate a linear fit in $M_{\pi}^{2}$ accounting for a dependence on the lattice spacing $a^{2}$ and interpolated in strange quark mass using $2 M_{K}^{2}-M_{\pi}^{2}$. The left plot of $f_{D}$ includes a simple linear fit as well as the chiral NLO fit for the ensembles with $M_{\pi}<450 \mathrm{MeV}$.

erties of these lattices enable successful fits of quantities to NLO and NNLO ChPT. Measurements are still in progress and the precision of the decay constants and LECs should be improved with better statistics and the use of stochastic noise sources.

The fine lattice spacings allow us to compute charmed masses and decay constants with small dependence upon $a$. We plan to produce results with quarks heavier than the charm mass to investigate the lattice spacing dependence for heavy domain wall quarks. If the dependence continues to remain small it may be possible to extrapolate to $B$ physics.

Numerical simulations are performed on the IBM System Blue Gene Solution at High Energy Accelerator Research Organization (KEK) under a support of its Large Scale Simulation Program (No. 13/14-04, 14/15-10). We thank P. Boyle for helping in the optimization of the code for BGQ. This work is supported in part by the Grant-in-Aid of the Japanese Ministry of Education (No. 
26400259, 26247043, and 15K05065) and the SPIRE (Strategic Program for Innovative Research) Field5 project.

\section{References}

[1] R. C. Brower, H. Neff, and K. Orginos, The Möbius Domain Wall Fermion Algorithm, arXiv:1206.5214.

[2] J. Noaki, S. Aoki, G. Cossu, S. Fukaya, H. Hashimoto, and T. Kaneko, Fine lattice simulations with chirally symmetric fermions, POS (LATTICE 2013) 263 (2014).

[3] C. Morningstar and M. Peardon, Analytic smearing of SU(3) link variables in lattice QCD, Phys. Rev. D 69 (Mar, 2004) 054501.

[4] S. Hashimoto, S. Aoki, G. Cossu, H. Fukaya, T. Kaneko, J. Noaki, and P. A. Boyle, Residual mass in five-dimesional fermion formulations, POS (LATTICE 2013) 431 (2014).

[5] S. BorsÃąnyi, S. DÃijrr, Z. Fodor, C. Hoelbling, S. Katz, S. Krieg, T. Kurth, L. Lellouch, T. Lippert, C. McNeile, and K. SzabÃş, High-precision scale setting in lattice QCD, Journal of High Energy Physics 2012 (2012), no. 9.

[6] G. Cossu, J. Noaki, S. Hashimoto, T. Kaneko, H. Fukaya, P. Boyle, and J. Doi, JLQCD IroIro++ lattice code on BG/Q, POS (LATTICE 2013) 482 (2014).

[7] S. Dürr, Z. Fodor, C. Hoelbling, S. Krieg, T. Kurth, L. Lellouch, T. Lippert, R. Malak, T. Métivet, A. Portelli, A. Sastre, and K. Szabó, Lattice QCD at the physical point meets su(2) chiral perturbation theory, Phys. Rev. D 90 (Dec, 2014) 114504.

[8] M. Tomii, G. Cossu, B. Fahy, H. Fukaya, S. Hashimoto, and J. Noaki, Analysis of short-distance current correlators using OPE, POS (LATTICE 2015) 266 (2015).

[9] J. Bijnens, G. Colangelo, and P. Talavera, The vector and scalar form factors of the pion to two loops, Journal of High Energy Physics 1998 (1998), no. 05014.

[10] K. Nakayama, B. Fahy, and S. Hashimoto, Charmonium current-current correlators with mobius domain-wall fermions, POS (LATTICE 2015) 267 (2015).

[11] B. Grinstein, E. Jenkins, A. V. Manohar, M. J. Savage, and M. B. Wise, Chiral perturbation theory for $f_{D_{s}} / f_{d}$ and $b_{B_{s}} / b_{b}$, Nuclear Physics B 380 (1992) 369. 